\title{
Applications of Microbial Biopolymers in Display Technology
}

\author{
Fabian B. H. Rehm, Katrin Grage, and Bernd H. A. Rehm
}

\section{Contents}

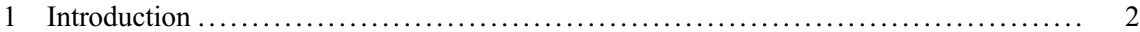

2 Polyhydroxyalkanoates .................................................... 2

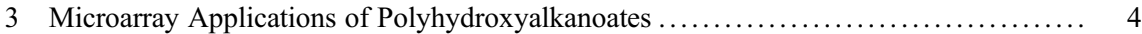

4 Tissue Culture/Engineering Applications of Polyhydroxyalkanoates .................. 7

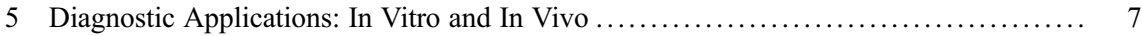

5.1 In Vitro Diagnostics ................................................ 7

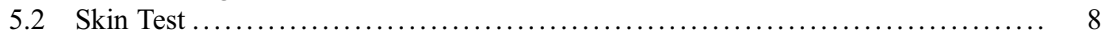

6 Particulate Antigen Delivery Systems ................................... 10

7 Recombinant Protein Production via Display on Polyhydroxyalkanoate Inclusions ....... 10

8 Affinity Bioseparation Applications Using Display of Binding Domains .............. 13

9 Research Needs ....................................................... 14

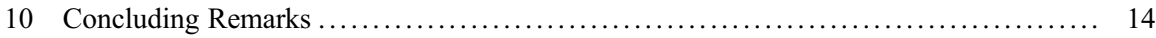

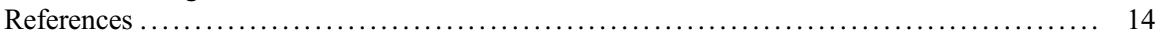

\begin{abstract}
Microorganisms produce a variety of different polymers such as polyamides, polysaccharides, and polyesters. The polyesters, the polyhydroxyalkanoates (PHAs), are the most extensively studied polymers in regard to their use in display technology. The material properties of bacterial PHAs in combination with their biocompatibility and biodegradability make them attractive substrates for use in display technology applications. By translationally fusing bioactive molecules to a gene encoding a PHA-binding domain, the appropriate functionalization for a given application can be achieved such that the need for chemical immobilization is circumvented. By separately extracting and processing the biopolymer, using it to coat a surface, and then treating this surface with the
\end{abstract}

F. Rehm • K. Grage • B.H.A. Rehm $(\bowtie)$

Institute of Fundamental Sciences, Massey University, Palmerston North, New Zealand

e-mail: b.rehm@massey.ac.nz 
fusion proteins, surface functionalization for immunodiagnostic microarray or tissue engineering applications can be accomplished. Conversely, by expressing the fusion protein directly in the PHA-producing organisms, one-step production of functionalized beads can be achieved. Such beads have been demonstrated in diverse applications, including fluorescence-activated cell sorting, enzyme-linked immunosorbent assays, microarrays, diagnostic skin test for tuberculosis, vaccines, protein purification, and affinity bioseparation.

\section{Introduction}

The display of biologically active molecules is utilized for a range of applications such as diagnostics, biosensing, and microarray technologies. The substrate on which such display takes place is greatly deterministic of functionality and applicability. Common, well-established techniques include display on cell surfaces, ribosomes, and phage particles (Lee et al. 2003; Zahnd et al. 2007; Rakonjac et al. 2011). The use of microbial biopolymers as substrates has more recently been revealed in a variety of contexts. Although bacteria can produce a range of polymers, only a few of them have been considered for display technologies (Fig. 1). Bacterial cellulose, which exhibits various properties superior to plant-based cellulose with respect to display technology applications, has been limited to enzyme, bacterial cell and fungi immobilization (Wu and Lia 2008; Ullah et al. 2016). In contrast, bacterial polyhydroxyalkanoates (PHAs) have been extensively investigated as substrates for display technology applications, and thus will be the focus of this chapter.

\section{Polyhydroxyalkanoates}

PHAs are biopolyesters which serve as carbon and energy storage materials in a range of bacteria and archaea (Lenz and Marchessault 2005; Rehm 2010). During excess carbon availability, they are stockpiled as the amorphous core of PHA inclusions, surrounded by structural proteins (phasins), PHA metabolism-associated enzymes, and regulator proteins (Grage et al. 2009; Jendrossek 2009). Critical enzymes for PHA synthesis and inclusion assembly are the PHA synthases (such as PhaC) which catalyze the stereoselective conversion of the activated precursor (R)-3-hydroxyacyl-CoA (Rehm 2003). These CoA thioesters, depending on their carbon chain length, are synthesized from intermediates of fatty acid metabolism or directly from acetyl-CoA to polyoxoesters with the simultaneous release of coenzyme A (Steinbuchel et al. 1993; Rehm 2006) (Fig. 2). Unlike the other hydrophobically interacting PHA inclusion surface proteins, the PHA synthase remains covalently linked to the PHA inclusion core (Hezayen et al. 2002; Peters and Rehm 2006).

Although PHAs are all hydrophobic and water insoluble, they can drastically vary in composition and thus physical properties. Melting points can range from $50{ }^{\circ} \mathrm{C}$ to 


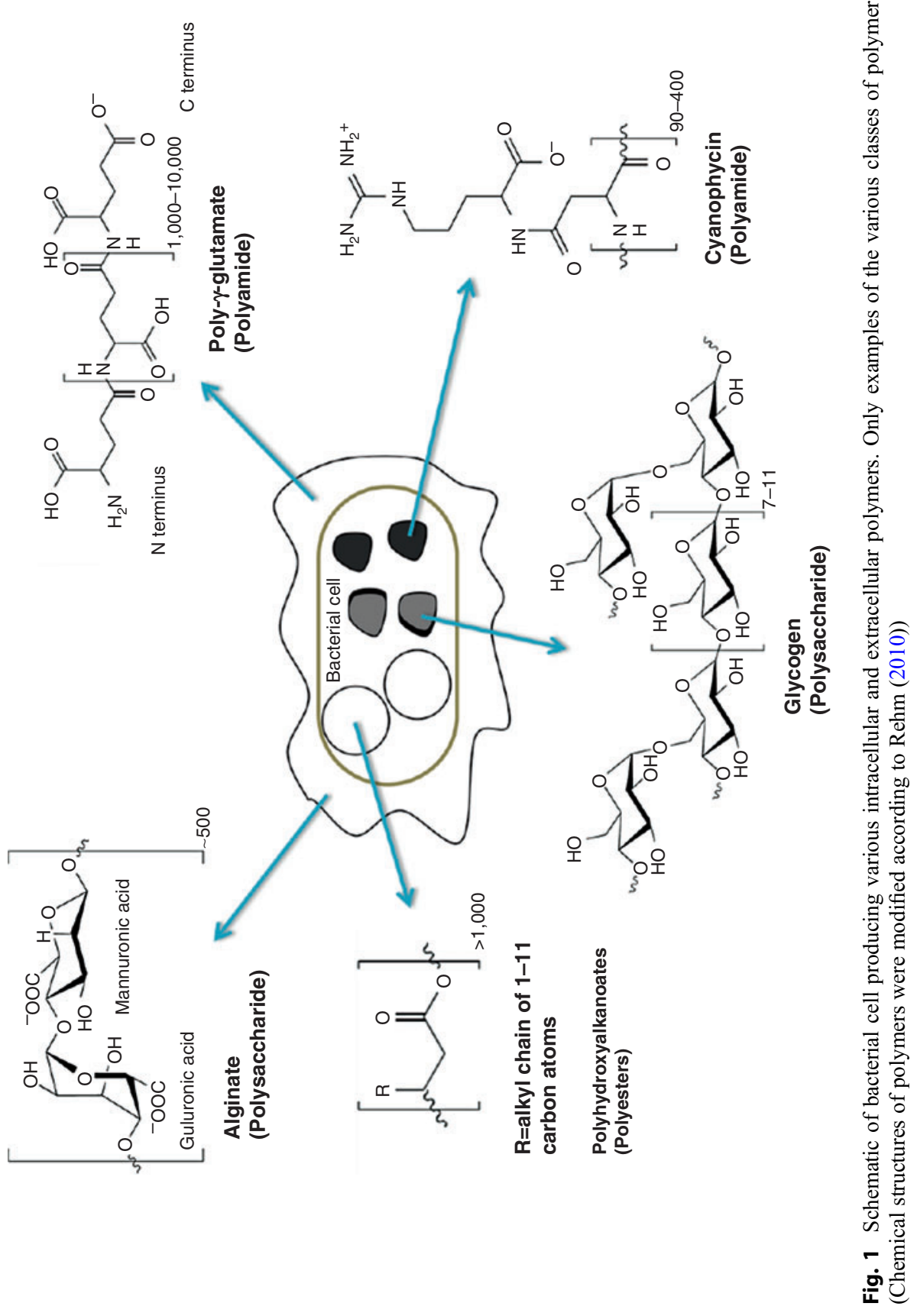




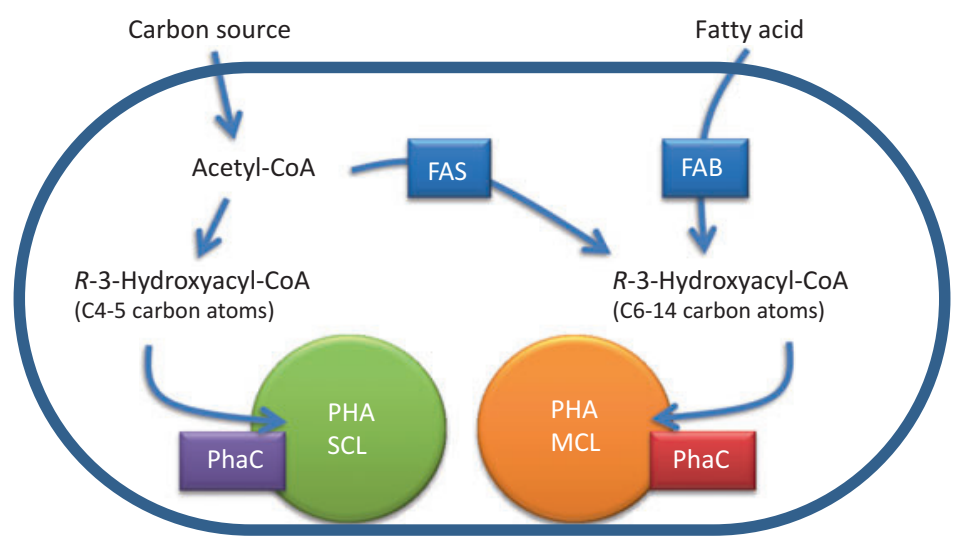

Fig. 2 Major steps in polyhydroxyalkanoate (PHA) biosynthesis. Arrows indicate enzyme catalyzed conversion reactions. FAS fatty acid synthesis, $F A B$ fatty acid beta-oxidation, PhaC PHA synthase, $S C L$ short chain-length, $M C L$ medium chain-length

$180{ }^{\circ} \mathrm{C}$ and crystallinity can range from $30 \%$ to $70 \%$, which is largely determined by monomer composition (Rehm 2010). As such, PHAs have been classified on the basis of monomer chain length. Medium-chain-length PHAs (MCL, C6-C14) are naturally produced primarily by pseudomonads, whereas short-chain-length PHAs (SCL, C3-C5) production is more widespread throughout bacteria and archaea (Anderson et al. 1990) (Fig. 2). While the common laboratory bacterium Escherichia coli does not naturally accumulate PHAs, it becomes a competent PHA producer upon introduction of the appropriate PHA biosynthesis genes (Schubert et al. 1988; Lee et al. 1994). The intracellular PHA inclusions may be isolated for polymer extraction and purification for processing into various materials, or maintained as functional shell-core beads (Fig. 3). The latter requires engineering of proteins attached to the PHA core in order to obtain functionality.

\section{Microarray Applications of Polyhydroxyalkanoates}

The PHA material properties enable film coating of solid surfaces suitable for microarray applications. Indeed, by exploiting the ability of PHA-associated proteins to specifically bind PHAs, and thereby overcoming specificity- or orientation-associated issues, PHA substrates for immobilization have been demonstrated as attractive for such applications.

The first relevant example of PHA as a protein micropatterning substrate was described in a study by Park and colleagues (2005). Poly(3-hydroxybutyrate) $(\mathrm{P}(3 \mathrm{HB}))$ and poly(3-hydroxybutyrate-co-3-hydroxyhexanoate) were independently produced, purified, and used to spin-coat glass substrate, producing PHA films. Subsequently, enhanced green fluorescent protein and red fluorescent protein, fused with the hydrophobic side chain-interacting substrate binding domain (SBD) 


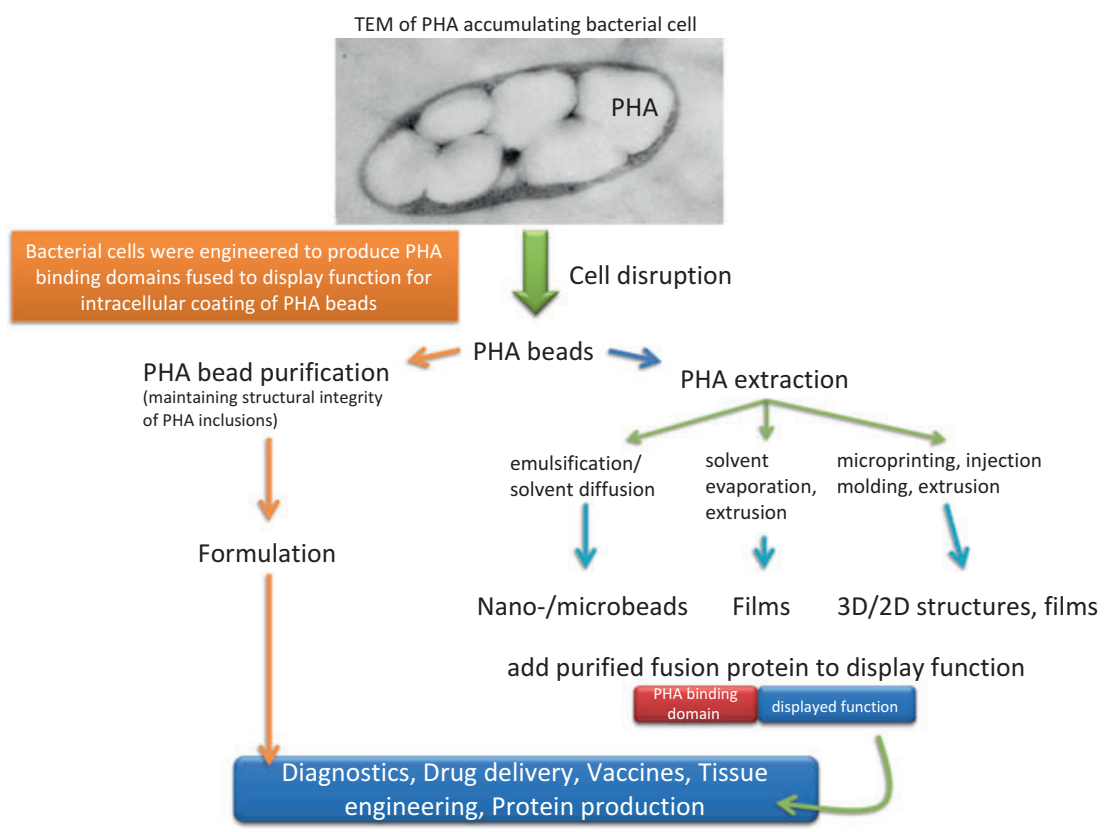

Fig. 3 Polyhydroxyalkanoate processing for display technologies. Comparison of in vivo and in vitro approaches towards the production of PHA-based material implementing display technologies

of a PHA depolymerase, were micropatterned onto the film using microcontact printing. Confocal fluorescent microscopy clearly revealed the printed patterns after several wash steps and surface plasmon resonance spectroscopy using antigreen fluorescent protein polyclonal antibody further confirmed this specific fusion protein immobilization, additionally demonstrating the system as capable for examining protein-protein interactions.

Spurred on by these encouraging results, a further study aimed to demonstrate the possibilities of this system for protein microarray development centered on immunodiagnostic applications (Park et al. 2006). PHA depolymerase SBD fusions with the single-chain antibody variable region (scFv) against hepatitis B virus (HBV) preS2 surface protein as well as the severe acute respiratory syndrome coronavirus envelope protein ( $\mathrm{SCVe}$ ) were produced and microspotted onto $\mathrm{P}(3 \mathrm{HB})$ films. Fluorescence-labelled HBV antigen and anti-SCVe antibody were then used to detect the interactions on the films. Fluorescence signals were detected only at the corresponding microspotted regions, indicating high affinity and selectivity and thus indicating the technology as appropriate for use in immunodiagnostics.

Subsequent investigation into whether such a platform could be applicable for clinical pathogen detection via immobilized DNA-protein complexes was undertaken by Park et al. (2009) (Fig. 4). Pathogen-specific (Acinetobacter baumannii, E. coli, Klebsiella pneuomoniae, and Pseudomonas aeruginosa) biotin-labelled 

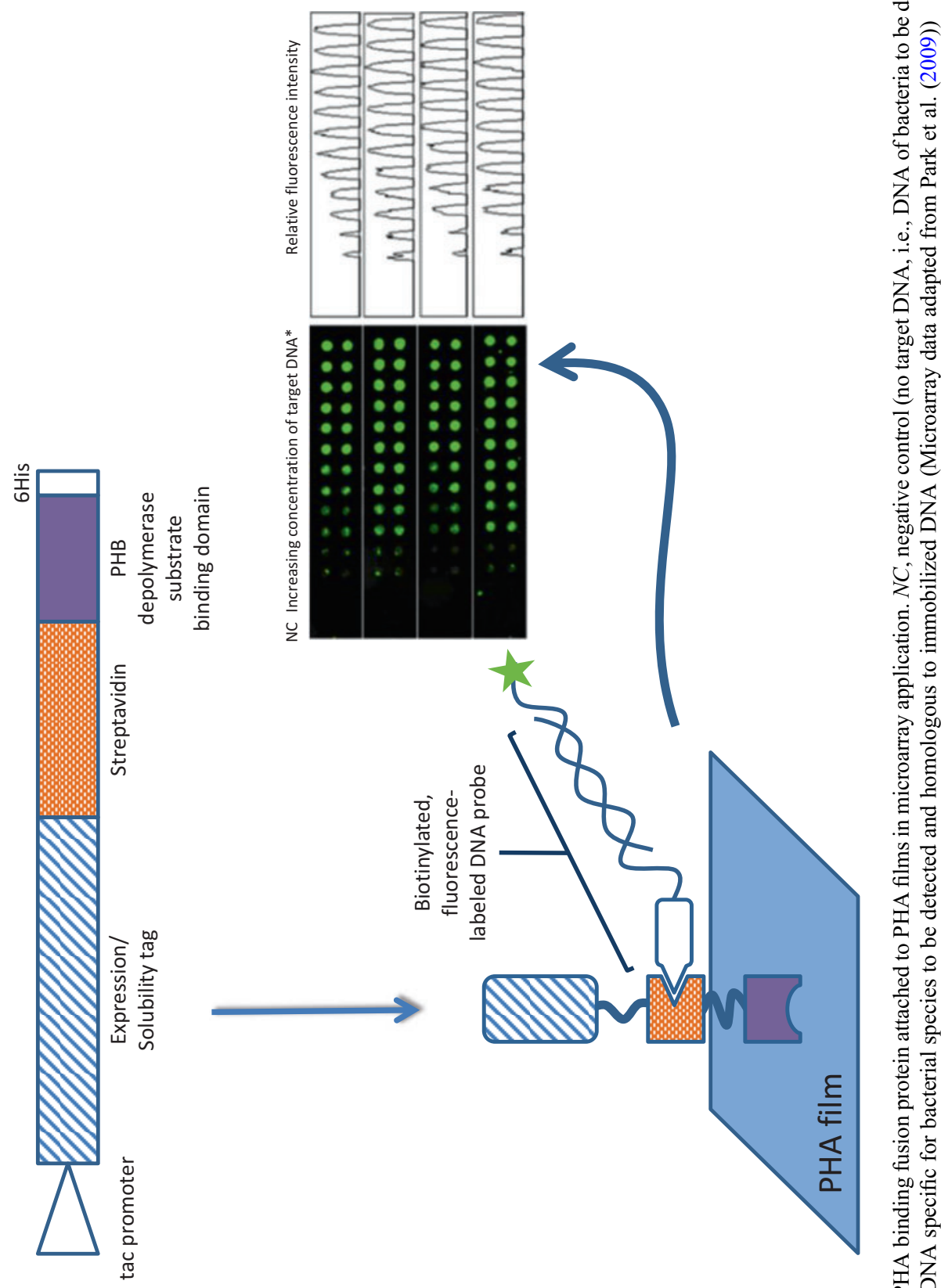

更莡

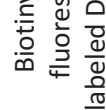

ब

惫

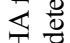

I

웅

요

过

ह

을

이유

跤

ह क

昰䒘

+ 
15-mer DNA probes immobilized to core streptavidin fused to the PHA depolymerase SBD were microspotted onto polyhdroxybutyrate (PHB)-coated slides. By hybridizing differentially fluorescently labelled target DNAs to each pathogen-specific probe simultaneous detection of the corresponding pathogens added to the slide was demonstrated, further evidencing the potential of PHAs for use in immunodiagnostic microarrays.

\section{$4 \quad$ Tissue Culture/Engineering Applications of Polyhydroxyalkanoates}

The diverse, adjustable material properties of PHAs in concert with their biodegradability, biocompatibility, noncarcinogenicity, and low cytotoxicity have driven their development in the area of biomaterials research (Ali and Jamil 2016). On the basis of PHA surface functionalization by addition of PHA-binding protein fusions, several approaches to enhancing tissue engineering and culturing techniques have been undertaken.

By translationally fusing the PHA-binding phasin, PhaP, to the cell adhesion motif Arg-Gly-Asp (RGD) and applying this fusion protein to coat PHA surfaces, the effect on fibroblast growth was investigated (Dong et al. 2010). Under serum-free conditions, confocal laser scanning microscopy in combination with cell counting assays revealed a significant cell attachment increase on the PhaP-RGD-coated PHA films relative to the PhaP- (nonfused) and noncoated films, in a manner that is unlikely to be attributable to changes in PHA surface topology. Further cell proliferation assays revealed increased fibroblast proliferation levels on PhaP-RGDcoated PHA films. Thus, PHA surface functionalization, using a well-known cell adhesion motif, was demonstrated using aqueous solutions, avoiding toxicity of chemical immobilization techniques. Continuing this pattern of investigation, similar increases in cell adhesion and cell proliferation of human vascular smooth muscle cells (HvSMCs) were demonstrated using the PHA repressor protein (PhaR) fused to the specific integrin ligand peptide (KQAGDV) (Dong et al. 2012), and, more recently, PhaP fused to RGD or the laminin-derived IKVAV peptides used to coat PHA films demonstrated enhanced neural stem cell (NSC) attachment, proliferation, and better neurite outgrowths, without effecting NSC differentiation (Xie et al. 2013).

\section{Diagnostic Applications: In Vitro and In Vivo}

\subsection{In Vitro Diagnostics}

Fluorescence activated cell sorting (FACS) is a qualitative and quantitative technique used in biomolecule detection for in vitro diagnostics. Generally, antigen-displaying beads are used to bind antibodies which are then detected via fluorescent-signaling secondary antibodies when the bead suspension passes through the FACS machine 
flow cell. Current bead preparation techniques involve tedious antigen purification and chemical crosslinking. Therefore, more cost-effective, reliable means of producing antigen-displaying beads with comparable detection efficacy could greatly impact the accessibility of FACS. A series of studies have shown that one path towards such improvement may be via one-step production of surface protein engineered PHA beads.

The first demonstration of in vivo-produced PHA inclusions for FACS applications was described by Bäckström and coworkers (Bäckström et al. 2007). The authors generated fusions between either interleukin-2 (IL2) or the myelin oligodendrocyte glycoprotein (MOG) and the C- or N-terminus of PhaP via an enterokinase (specific protease) recognition site providing linker. Subsequently, the hybrid genes were expressed in E. coli and the PHA granules extracted. The resultant antigen-displaying PHA beads were then used for FACS using the corresponding fluorescently labelled monoclonal antibody which showed significant and specific antibody binding. Enterokinase treatment reversed this recognition indicating removal of the fusion partners/antigens. Finally, sera from mice immunized with MOG or ovalbumin (as a negative control) were analyzed using the MOG-displaying beads and FACS. Again, high specificity and sensitivity (antibody detection in sera diluted 1:100,000) were demonstrated, providing strong support for application of engineered PHA beads in FACS (Fig. 5). A subsequent investigation explored C-terminal PhaC-streptavidin fusions (Peters and Rehm 2008). The remarkably high streptavidin-biotin binding affinity has given rise to its use in range of biotechnological applications, thus making it a great candidate for further demonstrating the applicability of PHA beads in FACS. The study revealed a biotin binding capacity of $61 \mathrm{ng}$ per $\mu \mathrm{g}$ of bead protein and demonstrated the detection of goat polyclonal biotinylated IgG in FACS using a secondary conjugated antibody. Most recently, PHA inclusions with GFP and MOG fused to PhaP or PhaC, such that each granule is simultaneously displaying two protein-based functionalities, were examined in the context of FACS, providing proof-of-concept for the biotechnological application of bifunctional PHA beads which may extend beyond FACS (Atwood and Rehm 2009).

The enzyme-linked immunosorbent assay (ELISA) is based on antigen-antibody binding on solid support either detecting antigen or antibody in samples. In vivo assembled engineered PHA inclusions displaying antigens or specific binding domains as described above were directly used to coat ELISA plates. Respective ELISAs showed specific and sensitive detection of the corresponding antibody or antigen confirming the applicability of bioengineered PHA beads in ELISA (Peters and Rehm 2008; Atwood and Rehm 2009; Parlane et al. 2009).

\subsection{Skin Test}

One in vivo-produced PHA bead-based approach to in vivo diagnosis is a skin test for the detection of bovine tuberculosis (TB) (Chen et al. 2014). The major disadvantage of the currently widespread tuberculin skin test is the lack of detection 


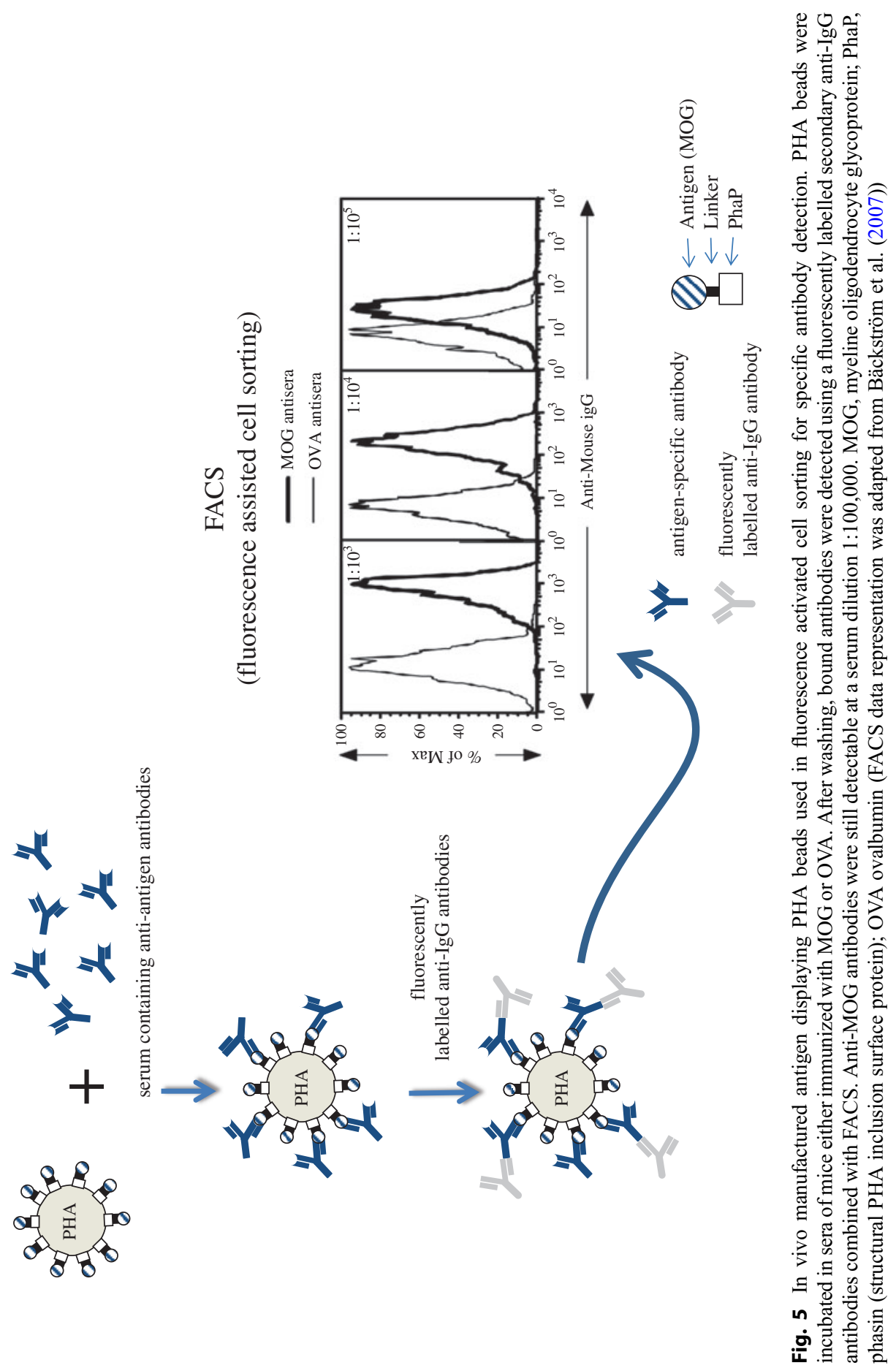


specificity for pathogenic Mycobacterium tuberculosis complex members, that is, cattle exposed to nonpathogenic, environmental mycobacteria may result in falsepositives. Thus, with the aims of improving specificity and lowering production cost, an alternative test was developed. Three immunodominant TB antigens, which are absent in most environmental mycobacteria, were fused to $\mathrm{PhaC}$ mediating production of triple-antigen-displaying PHA beads. These granules showed in vitro increased reactivity with antigen-specific TB antibodies when compared with granules displaying only one antigen. Assessment of triple-antigen-displaying PHA beads in the skin test (in vivo) showed specificity as all cattle experimentally infected with Mycobacterium bovis were detected while no false-positive reactions in cattle previously exposed to environmental mycobacteria were observed. A fourth antigen was added to the triple-antigen-displaying PHA beads to boost skin test sensitivity (Parlane et al. 2016). Dose response studies showed that very low amounts of mycobacterial antigens $(\geq 0.1 \mu \mathrm{g})$ were already sufficient for the skin test suggesting greatly increased immunogenicity of antigen-displaying beads versus soluble antigens. Hence, it was demonstrated that antigen-displaying PHA beads mediate an antigen-specific immune reaction upon injection into the skin, that is, serve as specific immune response stimulating antigen delivery systems.

\section{Particulate Antigen Delivery Systems}

The concept of in vivo assembly of nano-/microsized PHA beads displaying diseasespecific antigen as particulate vaccines was investigated. Since PHAs are considered as biomaterials, when produced coated with pathogen-specific antigens they might serve as safe and efficient particulate vaccine. In general, the nano-/microsized particulate nature of a vaccine mimics the dimensions of a pathogen which boosts immunogenicity via enhanced uptake by antigen presenting cells (APC) (Shah et al. 2014). Immunodominant antigens from pathogens such as HCV and M. tuberculosis were displayed on PHA beads using PHA synthase engineering (Parlane et al. 2009, 2011). Purified antigen-coated PHA beads were injected into mice which resulted in strong and specific immune responses mediating protective immunity as assessed by challenge of vaccinated animals with the pathogen (Parlane et al. 2012; MartinezDonato et al. 2016) (Fig. 6). It is noteworthy that these PHA bead-based vaccines stimulated both a humoral antibody (Th2) and cell-mediated (Th1) immune response, the latter being particularly relevant to protect against intracellular pathogens and is more challenging to achieve.

\section{$7 \quad$ Recombinant Protein Production via Display on Polyhydroxyalkanoate Inclusions}

Purification of recombinantly produced proteins, generally with the target protein fused to an affinity tag, can be a time-consuming and costly process since it often requires multiple chromatography steps which all need to be individually optimized, 


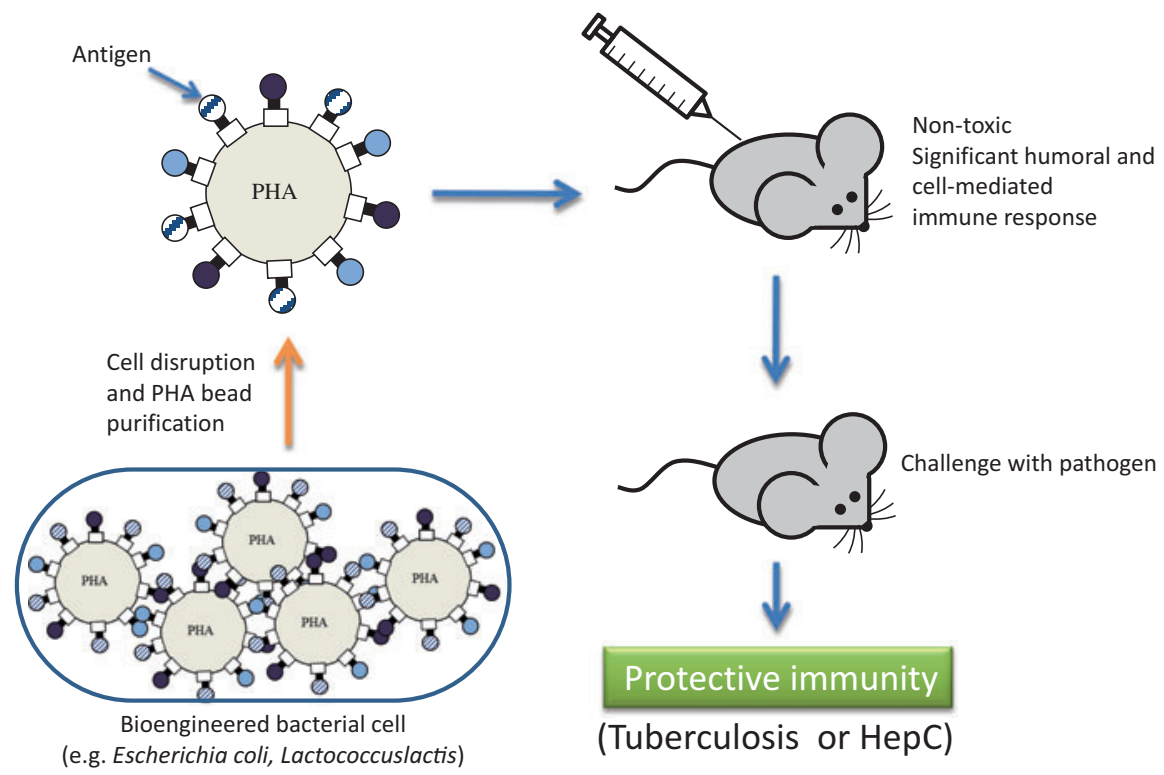

Fig. 6 PHA inclusions displaying disease-specific antigens as particulate vaccines

and additionally require cleavage and removal of the tag. Over the past 10 years, various approaches have been undertaken to make use of PHA and PHA-associated proteins for the development of alternative, simple and cost-effective protein purification systems. The general principle that most of these studies have followed is to produce the target protein as a translational fusion to a PHA inclusion-associated protein in cells that also make PHA and to then copurify the protein with the PHA beads. This can be done in a host that naturally produces PHA or in an organism that has been genetically engineered to make PHA (e.g., E. coli). Different methods have then been applied to release the target protein from the beads. Early examples used the phasin protein PhaP as an affinity tag and inducible self-cleaving inteins inserted between PhaP and the target protein to release the protein of interest. This method was applied by Barnard and coworkers to purify green-fluorescent protein (GFP) and $\beta$-galactosidase (LacZ) from Ralstonia eutropha, a natural PHB producer (Barnard et al. 2005). GFP and LacZ could be successfully purified fused to either end of PhaP using a thiol-inducible intein. Similarly, several proteins (maltose-binding protein (MBP), LacZ, chloramphenicol acetyltransferase (CAT), and NusA) were tagged with PhaP and purified from recombinant $E$. coli with the help of a $\mathrm{pH}$-inducible intein (Banki et al. 2005).

To strengthen the binding of the fusion protein to the bead surface to the point where significant leakage during the purification process could be avoided, multiple (two or three) phasins were used as a tag. Geng and coworkers chose a protein which is generally difficult to produce in bacteria due to the presence of several disulfide bonds (Geng et al. 2010). They fused recombinant human tissue plasminogen 
activator (rPA), a truncated version of tissue plasminogen activator, to $\mathrm{PhaP}$ and inserted a thrombin cleavage site as a linker. Active rPA could be released from isolated beads by thrombin treatment.

In another study which focused on diagnostic applications (aforementioned) the MOG and interleukin-2 were produced as PhaP-fusions containing an enterokinase recognition site (Bäckström et al. 2007). The successful removal of MOG or IL2, respectively, after enterokinase treatment was monitored by FACS analysis.

A slightly different approach to PHA-based protein purification is based on production of fusion proteins separate to PHA extraction and processing into beads. Various target proteins were fused to different granule-associated proteins such as PhaP or the regulatory protein PhaR, the fusion proteins recombinantly produced in E. coli and crude cell lysates incubated with beads processed from extracted PHA (Wang et al. 2008, Zhang et al. 2010). Release of the target protein was also achieved via intein-mediated self-cleavage. While this method is more labor-intensive than producing PHA and proteins in the same cell, advantageous might be the possibility to produce the tagged target protein in any host organism including eukaryotes. A simplified approach used the N-terminal part of PhaF to anchor a target protein to PHA beads in vivo, followed by bead isolation and release of the target protein (i.e., the entire fusion of target protein and $\mathrm{PhaP}$ ) from the beads by detergent treatment (Moldes et al. 2004).

However, a general drawback of using PhaP (or PhaR) as a tag for protein purification is that these proteins are only attached to the PHA inclusion surface via hydrophobic interactions, so there is a risk that the fusion protein could detach during either the bead purification process or the tag cleavage process resulting in loss of target protein. In the natural system, phasins have the advantage of being the predominant protein on the surface of PHA inclusions (Wieczorek et al. 1995); however, in a recombinant system, proteins such as the PHA synthase can be overproduced to achieve a high density at the inclusion surface (Brockelbank et al. 2006; Mifune et al. 2009).

Grage et al. harnessed the covalent attachment of the PHA synthase to PHA inclusions by translationally fusing HcRed or an anti- $\beta$-galactosidase single-chain antibody fragment (scFv) (Martineau et al. 1998), separated by an enterokinase recognition site (Grage et al. 2011). Both target proteins were successfully released from purified beads by enterokinase treatment; however, cleavage efficiency was relatively low. In an attempt to find a robust and inexpensive auto-processing module, Hay and coworkers used a modified soluble form of the cell surface sortase transpeptidase A (SrtA) from Staphylococcus aureus which had been engineered to self-cleave in the presence of $\mathrm{Ca}^{2+}$ (Mao 2004; Hay et al. 2015a). SrtA and the target protein were fused to the C-terminus of PhaC using an extended linker (Jahns and Rehm 2009). Using this technique, GFP, MBP, and antigen RV1626 from Mycobacterium tuberculosis could be released from isolated PHA beads at a high yield and purity (e.g., $6 \mathrm{mg} / 1$ of soluble GFP at a purity of about 98\%) (Hay et al. 2015a). 


\section{$8 \quad$ Affinity Bioseparation Applications Using Display of Binding Domains}

It may not always be feasible or desirable to produce the target protein fused to a bead-associated protein (and to coproduce it with the beads/resin). Hence PHA beads have also been engineered to serve as affinity resins for bioseparation, generally exploiting the possibility of densely displaying binding domains on nano-/microbeads. These resins can be produced in one step by fusing the binding domain of choice to the bead-associated protein and then isolating these functionalized beads from the production host (similar to the protein purification approach described above). The first example of this was the immunoglobulin $G$ (IgG) binding ZZ domain of Staphylococcus aureus protein A translationally fused to $\mathrm{PhaC}$ (Brockelbank et al. 2006). The resulting ZZ domain displaying beads were isolated from $E$. coli and successfully purified $\mathrm{IgG}$ from human serum and mouse hybridoma supernatants, with purity and yield comparable to commercially available protein A sepharose (Brockelbank et al. 2006; Lewis and Rehm 2009).

Further PHA bead-based resins assembled by engineering PhaC displayed streptavidin which bound various biotinylated compounds such as enzymes, antibodies, and DNA (Peters and Rehm 2008). Grage and Rehm were able to purify $\beta$-galactosidase from a mixture of proteins using anti-LacZ scFv immobilized to PHA beads (Grage and Rehm 2008). An endotoxin-removing resin was developed by producing human lipopolysaccharide-binding protein (hLBP) fused to PhaP in Pichia pastoris (Li et al. 2011). After secretion and recovery from the culture supernatant, the hLBP-PhaP fusion was incubated with beads processed from extracted PHA, and the resulting hLBP-beads were tested for their endotoxin removal abilities under a variety of conditions. According to the authors, the beads performed better than commercially available endotoxin-removing gels ( $\mathrm{Li}$ et al. 2011).

Recently, Hay and coworkers published a more extensive study that aimed at broadening the applicability of PHA bead affinity resins by identifying and testing several easily customizable affinity binding domains which they translationally fused to PhaC (Hay et al. 2015b). This study demonstrated that $\mathrm{V}_{\mathrm{HH}}$ domains from camelid antibodies, designed ankyrin repeat proteins (DARPins) and OB-folds (OBodies), could be densely displayed on PHA beads resulting in high affinity binding resins for purification of various target proteins. These binding domains were used to establish extensive libraries of variants enabling screening for binders specific for the target compound of interest (Binz et al. 2004; Harmsen and De Haard 2007; Stumpp et al. 2008; Steemson et al. 2014). PHA-based affinity resins showed a purification performance at least equal to current commercial offerings (Hay et al. 2015b). A recent surface topology study of the $R$. eutropha PhaC attached to PHA inclusions suggested new engineering strategies towards the development of PHA-based affinity resins with increased binding capacity via improved display (Hooks and Rehm 2015). This study identified several surface-exposed flexible regions of PhaC, which tolerated the insertion of the IgG-binding $\mathrm{ZZ}$ domain. One 
of the double $\mathrm{ZZ}$ domain insertions (i.e., $\mathrm{ZZ}$ inserted in two of the surface-exposed regions) showed greatly improved IgG binding capacity with some of the single insertions also showing improved IgG binding capacity when compared with terminal fusions. Overall, PhaC engineering such as $\mathrm{N}$ - or C-terminal fusions and/or insertions enabled efficient display of binding domains for interaction with target compounds resulting in purification performance suitable for application as bioseparation resin.

\section{$9 \quad$ Research Needs}

Microbial PHAs show great promise as polymers providing a support structure for display of a range of protein functions. Since PHAs can be composed of various constituent resulting in a diversity of material properties, it currently remains unexplored how these different PHAs perform in the context of anchoring binding domains for display. Additionally, research needs to address bioprocessing challenges to obtain PHAs of consistent structure and shape for improved implementation in various protein display applications.

\section{Concluding Remarks}

Although microorganisms are capable of producing a variety of polymers, the hydrophobic thermoplastic PHAs currently hold the greatest promise as support material to display protein functions. The PHA material properties allow processing into nano-/microbeads, films, and 3D structures, while PHA-related proteins provide specific PHA binding domains to anchor protein functions of interest. These approaches enabled implementation in microarray-based diagnostics as well as tissue engineering. Besides the binding of protein functions to isolated and processed PHA, recent research elucidated the concept of producing PHA inclusions within the bacterial cell already coated with desired protein functions. The applicability of these PHA beads as vaccines, in diagnostics and as bioseparation resin was demonstrated.

\section{References}

Ali I, Jamil N (2016) Polyhydroxyalkanoates: current applications in the medical field. Front Biol 11(1):19-27

Anderson AJ, Haywood GW, Dawes EA (1990) Biosynthesis and composition of bacterial poly (hydroxyalkanoates). Int J Biol Macromol 12(2):102-105

Atwood JA, Rehm BH (2009) Protein engineering towards biotechnological production of bifunctional polyester beads. Biotechnol Lett 31(1):131-137

Bäckström BT, Brockelbank JA, Rehm BH (2007) Recombinant Escherichia coli produces tailormade biopolyester granules for applications in fluorescence activated cell sorting: functional display of the mouse interleukin-2 and myelin oligodendrocyte glycoprotein. BMC Biotechnol $7: 3$ 
Banki MR, Gerngross TU, Wood DW (2005) Novel and economical purification of recombinant proteins: intein-mediated protein purification using in vivo polyhydroxybutyrate (PHB) matrix association. Protein Sci 14(6):1387-1395

Barnard GC, McCool JD, Wood DW, Gerngross TU (2005) Integrated recombinant protein expression and purification platform based on Ralstonia eutropha. Appl Environ Microbiol 71 (10):5735-5742

Binz HK, Amstutz P, Kohl A, Stumpp MT, Briand C, Forrer P, Grutter MG, Pluckthun A (2004) High-affinity binders selected from designed ankyrin repeat protein libraries. Nat Biotechnol 22 (5):575-582

Brockelbank JA, Peters V, Rehm BH (2006) Recombinant Escherichia coli strain produces a ZZ domain displaying biopolyester granules suitable for immunoglobulin G purification. Appl Environ Microbiol 72(11):7394-7397

Chen S, Parlane NA, Lee J, Wedlock DN, Buddle BM, Rehm BHA (2014) New skin test for detection of bovine tuberculosis on the basis of antigen-displaying polyester inclusions produced by recombinant Escherichia coli. Appl Environ Microbiol 80(8):2526-2535

Dong Y, Li P, Chen CB, Wang ZH, Ma P, Chen GQ (2010) The improvement of fibroblast growth on hydrophobic biopolyesters by coating with polyhydroxyalkanoate granule binding protein PhaP fused with cell adhesion motif RGD. Biomaterials 31(34):8921-8930

Dong CL, Li SY, Wang Y, Dong Y, Tang JZ, Chen JC, Chen GQ (2012) The cytocompatability of polyhydroxyalkanoates coated with a fusion protein of PHA repressor protein (PhaR) and Lys-Gln-Ala-Gly-Asp-Val (KQAGDV) polypeptide. Biomaterials 33(9):2593-2599

Geng Y, Wang S, Qi Q (2010) Expression of active recombinant human tissue-type plasminogen activator by using in vivo polyhydroxybutyrate granule display. Appl Environ Microbiol 76 (21):7226-7230

Grage K, Rehm BH (2008) In vivo production of scFv-displaying biopolymer beads using a selfassembly-promoting fusion partner. Bioconjug Chem 19(1):254-262

Grage K, Jahns AC, Parlane N, Palanisamy R, Rasiah IA, Atwood JA, Rehm BH (2009) Bacterial polyhydroxyalkanoate granules: biogenesis, structure, and potential use as nano-/micro-beads in biotechnological and biomedical applications. Biomacromolecules 10(4):660-669

Grage K, Peters V, Rehm BH (2011) Recombinant protein production by in vivo polymer inclusion display. Appl Environ Microbiol 77(18):6706-6709

Harmsen MM, De Haard HJ (2007) Properties, production, and applications of camelid singledomain antibody fragments. Appl Microbiol Biotechnol 77(1):13-22

Hay ID, Du J, Reyes PR, Rehm BH (2015a) In vivo polyester immobilized sortase for tagless protein purification. Microb Cell Factories 14(1):190

Hay ID, Du J, Burr N, Rehm BH (2015b) Bioengineering of bacteria to assemble custom-made polyester affinity resins. Appl Environ Microbiol 81(1):282-291

Hezayen FF, Steinbuchel A, Rehm BH (2002) Biochemical and enzymological properties of the polyhydroxybutyrate synthase from the extremely halophilic archaeon strain 56. Arch Biochem Biophys 403(2):284-291

Hooks DO, Rehm BHA (2015) Insights into the surface topology of polyhydroxyalkanoate synthase: self-assembly of functionalized inclusions. Appl Microbiol Biotechnol 99 (19):8045-8053

Jahns AC, Rehm BH (2009) Tolerance of the Ralstonia eutropha class I polyhydroxyalkanoate synthase for translational fusions to its $\mathrm{C}$ terminus reveals a new mode of functional display. Appl Environ Microbiol 75(17):5461-5466

Jendrossek D (2009) Polyhydroxyalkanoate granules are complex subcellular organelles (carbonosomes). J Bacteriol 191(10):3195-3202

Lee SY, Choi JH, Xu Z (2003) Microbial cell-surface display. Trends Biotechnol 21(1):45-52

Lee SY, Yim KS, Chang HN, Chang YK (1994) Construction of plasmids, estimation of plasmid stability, and use of stable plasmids for the production of poly(3-hydroxybutyric acid) by recombinant Escherichia coli. J Biotechnol 32(2):203-211 
Lenz RW, Marchessault RH (2005) Bacterial polyesters: biosynthesis, biodegradable plastics and biotechnology. Biomacromolecules 6(1):1-8

Lewis JG, Rehm BHA (2009) ZZ polyester beads: an efficient and simple method for purifying IgG from mouse hybridoma supernatants. J Immunol Methods 346(1-2):71-74

Li JA, Shang GG, You ML, Peng SW, Wang ZH, Wu HN, Chen GQ (2011) Endotoxin removing method based on lipopolysaccharide binding protein and polyhydroxyalkanoate binding protein PhaP. Biomacromolecules 12(3):602-608

Mao HY (2004) A self-cleavable sortase fusion for one-step purification of free recombinant proteins. Protein Expr Purif 37(1):253-263

Martineau P, Jones P, Winter G (1998) Expression of an antibody fragment at high levels in the bacterial cytoplasm. J Mol Biol 280(1):117-127

Martinez-Donato G, Piniella B, Aguilar D, Olivera S, Perez A, Castanedo Y, Alvarez-Lajonchere L, Duenas-Carrera S, Lee JW, Burr N, Gonzalez-Miro M, Rehm BH (2016) Protective T cell and antibody immune responses against hepatitis $\mathrm{C}$ virus achieved using a biopolyester-bead-based vaccine delivery system. Clin Vaccine Immunol 23(4):370-378

Mifune J, Grage K, Rehm BH (2009) Production of functionalized biopolyester granules by recombinant Lactococcus lactis. Appl Environ Microbiol 75(14):4668-4675

Moldes C, Garcia P, Garcia JL, Prieto MA (2004) In vivo immobilization of fusion proteins on bioplastics by the novel tag BioF. Appl Environ Microbiol 70(6):3205-3212

Park JP, Lee KB, Lee SJ, Park TJ, Kim MG, Chung BH, Lee ZW, Choi IS, Lee SY (2005) Micropatterning proteins on polyhydroxyalkanoate substrates by using the substrate binding domain as a fusion partner. Biotechnol Bioeng 92(2):160-165

Park TJ, Park JP, Lee SJ, Hong HJ, Lee SY (2006) Polyhydroxyalkanoate chip for the specific immobilization of recombinant proteins and its applications in immunodiagnostics. Biotechnol Bioprocess Eng 11(2):173-177

Park TJ, Yoo SM, Keum KC, Lee SY (2009) Microarray of DNA-protein complexes on poly-3hydroxybutyrate surface for pathogen detection. Anal Bioanal Chem 393(6-7):1639-1647

Parlane NA, Wedlock DN, Buddle BM, Rehm BH (2009) Bacterial polyester inclusions engineered to display vaccine candidate antigens for use as a novel class of safe and efficient vaccine delivery agents. Appl Environ Microbiol 75(24):7739-7744

Parlane NA, Grage K, Lee JW, Buddle BM, Denis M, Rehm BH (2011) Production of a particulate hepatitis $\mathrm{C}$ vaccine candidate by an engineered Lactococcus lactis strain. Appl Environ Microbiol 77(24):8516-8522

Parlane NA, Grage K, Mifune J, Basaraba RJ, Wedlock DN, Rehm BH, Buddle BM (2012) Vaccines displaying mycobacterial proteins on biopolyester beads stimulate cellular immunity and induce protection against tuberculosis. Clin Vaccine Immunol 19(1):37-44

Parlane NA, Chen S, Jones GJ, Vordermeier HM, Wedlock DN, Rehm BH, Buddle BM (2016) Display of antigens on polyester inclusions lowers the antigen concentration required for a bovine tuberculosis skin test. Clin Vaccine Immunol 23(1):19-26

Peters V, Rehm BH (2006) In vivo enzyme immobilization by use of engineered polyhydroxyalkanoate synthase. Appl Environ Microbiol 72(3):1777-1783

Peters V, Rehm BHA (2008) Protein engineering of streptavidin for in vivo assembly of streptavidin beads. J Biotechnol 134(3-4):266-274

Rakonjac J, Bennett NJ, Spagnuolo J, Gagic D, Russel M (2011) Filamentous bacteriophage: biology, phage display and nanotechnology applications. Curr Issues Mol Biol 13(2):51-76

Rehm BH (2003) Polyester synthases: natural catalysts for plastics. Biochem J 376(Pt 1):15-33

Rehm BH (2006) Genetics and biochemistry of polyhydroxyalkanoate granule self-assembly: the key role of polyester synthases. Biotechnol Lett 28(4):207-213

Rehm BH (2010) Bacterial polymers: biosynthesis, modifications and applications. Nat Rev Microbiol 8(8):578-592

Schubert P, Steinbuchel A, Schlegel HG (1988) Cloning of the Alcaligenes eutrophus genes for synthesis of poly-beta-hydroxybutyric acid (PHB) and synthesis of PHB in Escherichia coli. J Bacteriol 170(12):5837-5847 
Shah RR, O'Hagan DT, Amiji MM, Brito LA (2014) The impact of size on particulate vaccine adjuvants. Nanomedicine (Lond) 9(17):2671-2681

Steemson JD, Baake M, Rakonjac J, Arcus VL, Liddament MT (2014) Tracking molecular recognition at the atomic level with a new protein scaffold based on the OB-fold. PLoS One 9(1):e86050

Steinbuchel A, Hustede E, Liebergesell M, Pieper U, Timm A, Valentin H (1993) Molecular basis for biosynthesis and accumulation of polyhydroxyalkanoic acids in bacteria. FEMS Microbiol Rev 10(3-4):347-350

Stumpp MT, Binz HK, Amstutz P (2008) DARPins: a new generation of protein therapeutics. Drug Discov Today 13(15-16):695-701

Ullah H, Wahid F, Santos HA, Khan T (2016) Advances in biomedical and pharmaceutical applications of functional bacterial cellulose-based nanocomposites. Carbohydr Polym 150:330-352

Wang Z, Wu H, Chen J, Zhang J, Yao Y, Chen GQ (2008) A novel self-cleaving phasin tag for purification of recombinant proteins based on hydrophobic polyhydroxyalkanoate nanoparticles. Lab Chip 8(11):1957-1962

Wieczorek R, Pries A, Steinbuchel A, Mayer F (1995) Analysis of a 24-kilodalton protein associated with the polyhydroxyalkanoic acid granules in Alcaligenes eutrophus. J Bacteriol 177(9):2425-2435

Wu S-C, Lia Y-K (2008) Application of bacterial cellulose pellets in enzyme immobilization. J Mol Catal B Enzym 54(3-4):103-108

Xie H, Li J, Li L, Dong Y, Chen GQ, Chen KC (2013) Enhanced proliferation and differentiation of neural stem cells grown on PHA films coated with recombinant fusion proteins. Acta Biomater 9 (8):7845-7854

Zahnd C, Amstutz P, Pluckthun A (2007) Ribosome display: selecting and evolving proteins in vitro that specifically bind to a target. Nat Methods 4(3):269-279

Zhang S, Wang ZH, Chen GQ (2010) Microbial polyhydroxyalkanote synthesis repression protein PhaR as an affinity tag for recombinant protein purification. Microb Cell Factories 9:28 